\title{
A compact high-efficiency power divider/combiner based on quadruple-ridged waveguide
}

\author{
Yihong Zhou ${ }^{1 \mathrm{a})}$, Haiyang Wang ${ }^{1}$, Jiayin $\mathrm{Li}^{1}$, and Haiyan $\mathrm{Jin}^{2}$ \\ ${ }^{1}$ School of Physical Electronics, University of Electronic Science and Technology \\ of China, No. 4 Section 2 North Jianshe Road, Chengdu, 610054, China \\ ${ }^{2}$ School of Communication and Information Engineering, \\ University of Electronic Science and Technology of China, \\ No. 4 Section 2 North Jianshe Road, Chengdu, 611731, China \\ a)zhouyh@uestc.edu.cn
}

\begin{abstract}
A compact high-efficiency four-way divider/combiner based on quadruple-ridged waveguide is presented. This divider/combiner designed at $31-38 \mathrm{GHz}$ is mainly composed of the quadruple-ridged transition, which features four-way power divider/combiner as well as transition from waveguide to microstrip. The measured power-combining efficiency of this circuit at $34 \mathrm{GHz}$ is up to $91 \%$, and the combining efficiency is higher than $80 \%$ at 31-38 GHz.
\end{abstract}

Keywords: power divider/combiner, quadruple-ridged waveguide, powercombining efficiency

Classification: Microwave and millimeter-wave devices, circuits, and modules

\section{References}

[1] N. Escalera, W. Boger, P. Denisuk and J. Dobosz: IEEE MTT-S Microw. Symp. Dig. (2000) 561. DOI:10.1109/MWSYM.2000.861123

[2] P. G. Courtney, T. Thuan, C. Bartak, S. Behan and P. C. Jia: IEEE MTT-S Microw. Symp. Dig. (2010) 1396. DOI:10.1109/MWSYM.2010.5517620

[3] M. Oz: EUMA Europ. Microw. Conf. (1984) 317. DOI:10.1109/EUMA.1984. 333415

[4] M. Oz, B. D. Geller, P. K. Cline and I. Yogev: EUMA Europ. Microw. Conf. (1991) 1029. DOI:10.1109/EUMA.1991.336481

[5] Y. M. Ning, W. S. Jiang and Q. L. Zhu: IEEE IWS Intelnat. Wireles. Symp. (2013) 1. DOI:10.1109/IEEE-IWS.2013.6616748

[6] Y. M. Ning, J. W. Sun, J. X. Liu and W. S. Jiang: URSI GASS Gener. Assemb. and Scienc. Symp. (2014) 1. DOI:10.1109/URSIGASS.2014.6929534

[7] R. Kazemi and A. E. Fathy: IET Microw. Anten. Propag. 9 (2015) 648. DOI:10.1049/iet-map.2014.0480

[8] Y. H. Zhou, H. Y. Wang, J. Y. Li and H. Y. Jin: IEICE Electron. Express 12 (2015) 20150816. DOI:10.1587/elex.12.20150861

[9] H. W. Yao, A. Abdelmonem, J. F. Liang and K. A. Zaki: IEEE Trans. Microw. Theory Techn. 42 (1994) 2371. DOI:10.1109/22.339769 
[10] Y. H. Zhou, J. Y. Li, H. Y. Jin and H. Y. Wang: IEICE Trans. Electron. E93-C (2010) 1484. DOI:10.1587/transele.E93.C.1484

[11] M. P. David: Microwave Engineering (Wiley, New York, 2012) 4th ed. 258.

[12] Y. Zhou, H. X. Liu, E. Li, G. F. Guo and T. Yang: ICMMT Microw. and Millim. Wave Tech. Conf. (2010) 737. DOI:10.1109/ICMMT.2010.5525049

\section{Introduction}

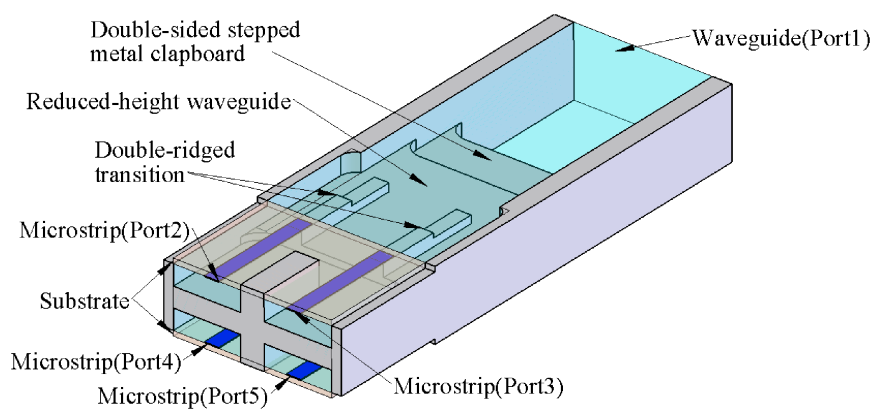

Fig. 1. Configuration of the four-way power divider/combiner based on quadruple-ridged waveguide

The power-combining technology is usually used to combine power from multiple devices to obtain the desired power level, which is very important in the development of high-power solid-state power amplifier $[1,2]$. Due to its characteristics of high power-combining efficiency and wide bandwidth, ridged waveguide is employed in some power divider/combiner designs [3, 4, 5, 6, 7, 8]. In [3], a doubleridged radial combiner with an $86 \%$ bandwidth in $\mathrm{C}$ and $\mathrm{X}$ band is proposed. In [4], another single-ridged radial combiner with a combining efficiency of up to $98 \%$ is proposed. In [5], a spatial power combiner based on broadband multi-way ridged waveguides is presented with a $29 \%$ bandwidth in Ka band. In [6], another spatial power combiner based on ridged waveguide is proposed, which can operate with a $24 \%$ bandwidth in V band. In [7], an eight-way compact combiner based on RSIW (ridge substrate integrated waveguide) is presented with a $65 \%$ bandwidth in $\mathrm{C}$ and $\mathrm{X}$ band. In [8], a compact four-way divider/combiner based on SIW (substrate integrated waveguide) and double-layer finline is also presented, and the measured power-combining efficiency of this circuit is $86 \%$ at $35.6 \mathrm{GHz}$ and higher than $75 \%$ at $29-40 \mathrm{GHz}$.

In this letter, based on quadruple-ridged waveguide in Ka band, a compact high-efficiency four-way power divider/combiner is presented, as shown in Fig. 1. In this design, the divider/combiner consists of quadruple-ridged waveguide transition, which mainly includes two compact parts: double-sided reduced-height waveguide and double-ridged transition. Double-sided reduced-height waveguide is constructed by inserting a double-sided stepped metal clapboard in the middle of a WR28 waveguide (see Fig. 1). Thus the WR28 waveguide can be transformed into two reduced-height waveguides with a very compact configuration. The double-ridged transition is achieved by placing two identical stepped ridges along the propagation direction of waveguide symmetrically, which can divide the input 
power into two microstrip branches as well as transform reduced-height waveguide into microstrips. Therefore, the whole structure of the power divider/combiner is very compact, and the functions of power division/combination and transition between different transmission lines can be realized within a standard WR28 waveguide. Due to low loss of ridged waveguide, the power-combining efficiency is also very high. The simulated and measured results of the proposed power divider/combiner are presented.

\section{Transition design}

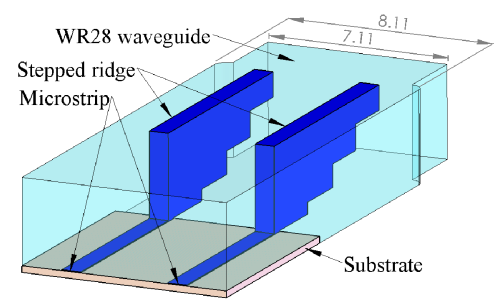

(a)

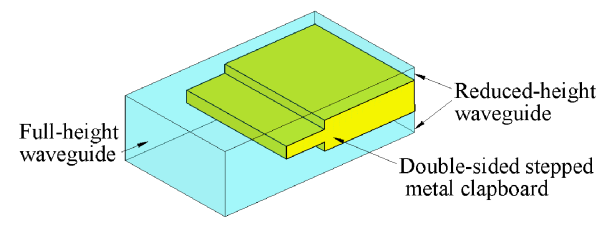

(b)

Fig. 2. (a) Double-ridged transition (b) Double-sided waveguide

\subsection{Double-ridged transition design}

Due to its low transmission loss and wide bandwidth, the ridged waveguide is often employed in low-loss microwave circuit. In order to be compatible with microstrip circuit, a typical ridged waveguide transition from waveguide to microstrip was studied by Huiwen Yao in [9]. For this transition, the propagation directions of waveguide and microstrip are consistent, which is very suitable for compact microwave circuit related to ridged waveguide and microstrip.

In [10], depending on the symmetry of TE10 mode of waveguide, the doublelayer finline can divide the input power into two branches. Due to the similarity of field distribution between finline and ridged waveguide, this power-dividing mechanism is also adaptable to the ridged waveguide. Instead of one stepped ridge in the transition in [9], two identical ones are placed along the propagation direction of waveguide symmetrically (see Fig. 2(a)). Due to the same electric-field orientation of waveguide and microstrip, TE10 mode of waveguide can be smoothly transformed into quasi-TEM mode of microstrip without any rotation of electric field. In addition, two identical ridges placed symmetrically can divide the input power into two equal branches, which realizes the function of power divider. In order to achieve a good match between waveguide and microstrips, Chebyshev quarter-wave broadband impedance matching network is used to determine the dimensions of each stepped ridge [11]. Thus, the double-ridged transition is deemed to be constructed by cascading multiple double-ridged waveguides with different ridge heights.

In addition, the spacing between two stepped ridges is also very important. Since the amplifier chip with high output power usually has a large dimension, a larger distance means more space to hold powerful amplifier chip. In order to achieve a large spacing, the cross-section of WR28 waveguide can be slightly widened in the broad side from $7.11 \mathrm{~mm}$ (the width of standard WR28 waveguide) 
to $8.11 \mathrm{~mm}$ (see Fig. 2(a)). However, the required double-ridged mode can not exist in a double-ridged transition with large ridge spacing. Therefore, the proper distance is a tradeoff of the above factors. In this letter, linear parameter-scanning method in HFSS is used to determine the spacing between two bridges. The scanning range of this spacing is from $0.8 \mathrm{~mm}$ to $6 \mathrm{~mm}$ with a step of $0.1 \mathrm{~mm}$, and an optimized spacing of $4.20 \mathrm{~mm}$ is selected from multiple parameter-scanning results.

\subsection{Double-sided reduced-height waveguide design}

For the compact microwave circuit related to waveguide, the reduced-height waveguide, whose height is less than that of full-height waveguide, is usually employed to reduce the volume of the circuit. Due to their similar TE10 modes, the reduced-height waveguide can simply be achieved by reducing the height of fullheight waveguide step by step. In the design of power divider/combiner, in order to make full use of the space in the height of the waveguide, two reduced-height waveguides are constructed back to back by inserting a double-sided stepped metal clapboard in the middle of a full-height waveguide (see Fig. 2(b)), and the input power from full-height waveguide can be divided into two reduced-height waveguide branches. Thus, a compact waveguide divider can be realized within a fullheight waveguide. In order to achieve a good matching, Chebyshev quarter-wave broadband impedance matching network is also used to determine the dimensions of each step [11].

\section{Divider design}

The divider (see Fig. 1) consists of double-sided reduced-height waveguide and two double-ridged transitions, and each reduced-height waveguide is cascaded to a double-ridged transition. All the four ridges constitute the quadruple-ridged waveguide, and each ridge branch is finally transformed into microstrip. Due to the high symmetry of the quadruple-ridge waveguide, the amplitude and phase different of each microstrip branch are coincident. In addition, due to the wideband essence of the double-ridge transition and double-sided reduced-height waveguide, the bandwidth of the cascaded divider should be broad.

For power-combining amplifier, the capability of heat sinking for amplifier chip is very important, which may limit the maximum output power of the amplifier chip. Therefore, in order to achieve a good heat sink, the microstrips are configured face to face (see Fig. 1), and heat produced by the amplifier chips assembled on the microstrip can rapidly be transferred outward.

\section{Simulated and experiment results}

For the proposed power divider, Rogers 5880 with a dielectric constant of 2.2 and a thickness of $0.25 \mathrm{~mm}$ was used as the substrate. In order to be compatible with $50 \Omega$ microstrip line, the width of ridge is $0.75 \mathrm{~mm}$ and the height of step B connected to microstrip line is $0.25 \mathrm{~mm}$ (see Fig. 3). Due to the up-down symmetry of this divider and the uniform characteristics of TE10 mode of WR28 waveguide in the height, the microstrip lines are deemed to be transformed from half-height wave- 
guide. Therefore, the input waveguide of each double-ridged transition is a reduced-height waveguide derived from half-height waveguide. In order to achieve a good matching between half-height waveguide and double-ridged transition, stepped metal clapboard and ridges are employed (see Fig. 3), which form a Chebyshev quarter-wave impedance matching network [11]. For this stepped matching network, the characteristic impedance $Z_{\mathrm{PV}}$ of each reduced-height waveguide and double-ridged waveguide is defined by the ratio of voltage $V$ and power [12], where $\mathrm{V}$ is the voltage along the center line of the waveguide and doubleridged waveguide. According to the definition of $Z_{\mathrm{PV}}$, the characteristic impedance and guide wavelength of each waveguide can be calculated directly in HFSS. The characteristic impedance ratio $\left(\mathrm{Z}_{1} / \mathrm{Z}_{0}\right)$ of half-height waveguide and double-ridged waveguide $B$ is about 5 . For Chebyshev matching network with $N=1,2,3$ transition steps, the relative bandwidth with a maximum reflection coefficient $\Gamma_{\mathrm{m}}=0.01$ is $1.5 \%, 19 \%, 45 \%$ respectively [11]. Therefore, a matching network with $\mathrm{N}=3$ transition steps can meet the design requirement of relative bandwidth of $20 \%$. As shown in Fig. 3, two transition steps are allocated to the stepped metal clapboard (reduced-height waveguide $\mathrm{D}$ and $\mathrm{E}$ ) and one is allocated to the stepped ridge (double-ridged waveguide $\mathrm{C}$ ). Given $\mathrm{N}=3, \Gamma_{\mathrm{m}}=0.01, \mathrm{Z}_{1} / \mathrm{Z}_{0}=5.2$, the characteristic impedance of each waveguide can be calculated by using the design method in [11], and these impedance values are used to determine the initialized dimensions of each waveguide in HFSS. The initialized ridge heights of doubleridged waveguide $\mathrm{B}$ and $\mathrm{C}$ and heights of reduced-height waveguide $\mathrm{D}$ and $\mathrm{E}$ are $\mathrm{h}_{\mathrm{B}}=0.25 \mathrm{~mm}, \mathrm{~h}_{\mathrm{C}}=0.47 \mathrm{~mm}, \mathrm{~h}_{\mathrm{D}}=0.85 \mathrm{~mm}$ and $\mathrm{h}_{\mathrm{E}}=1.3 \mathrm{~mm}$ respectively. The initialized lengths of each waveguide are $1_{B}=3 \mathrm{~mm}, 1_{C}=2.6 \mathrm{~mm}, 1_{D}=$ $2.8 \mathrm{~mm}$ and $l_{\mathrm{E}}=2.8 \mathrm{~mm}$ respectively, which are quarter guide wavelength of each waveguide. Based on these initialized dimensions, this divider was simulated and optimized in HFSS to achieve a good matching, and the structure of the divider with its final dimensions is shown in Fig. 3.

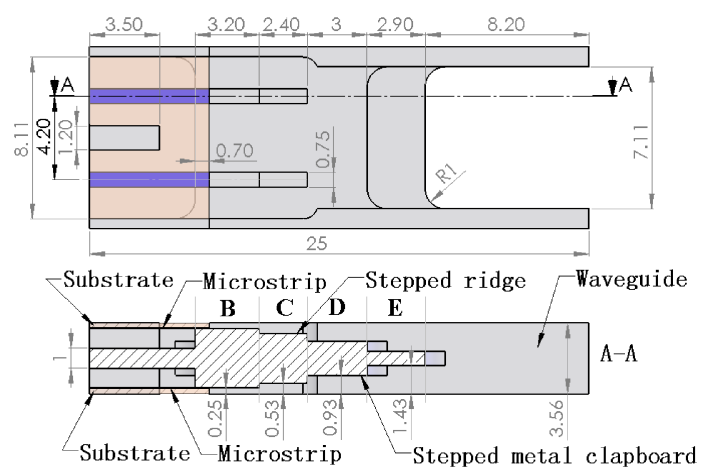

Fig. 3. The four-way divider based on quadruple-ridged waveguide 
passive divider/combiner circuit was built by placing two identical dividing circuits back to back (Fig. 5(a)), and the left and right parts of the power divider/combiner act as the role of heat sink as well as broad sides of the waveguide. The measured results of the power divider/combiner show a good agreement with the simulated results (Fig. 5(b)). The measured minimum insertion loss of $0.8 \mathrm{~dB}$ is achieved with a return loss of $25 \mathrm{~dB}$ at $34 \mathrm{GHz}$, which indicates a maximum power-combining efficiency of $91 \%$ for the combining circuit. In addition, the measured powercombining efficiency is higher than $80 \%$ at $31-38 \mathrm{GHz}$, which is higher than that of the combiner in [8] due to less transmission loss of ridged-waveguide than SIW. For the combiner, the increased insertion loss compared with the simulated one is most likely due to the transmission losses of ridged waveguide and microstrip lines and the mismatch of microstrip branches.

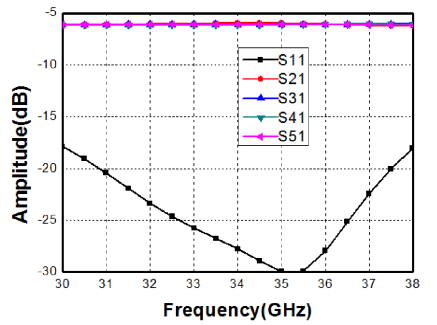

(a)

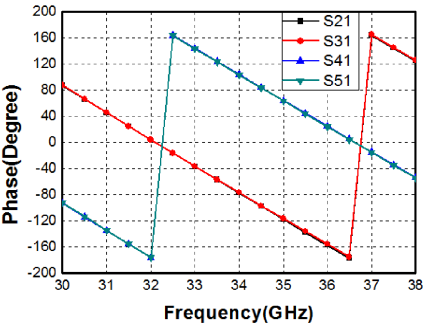

(b)

Fig. 4. Simulated results of the divider (a) Magnitude (b) Phase

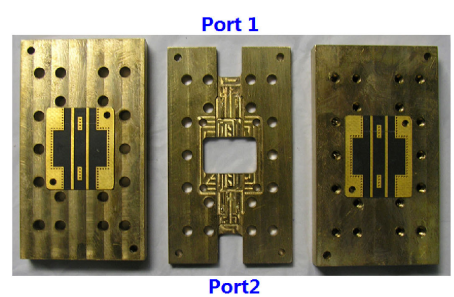

(a)

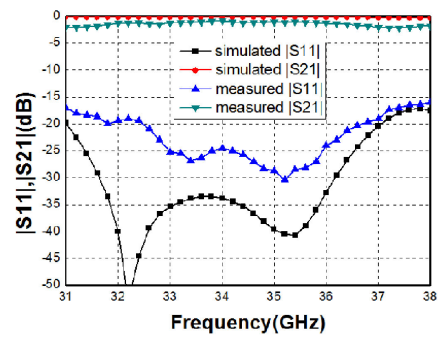

(b)

Fig. 5. (a) The prototype of the divider/combiner (b) The simulated and measured results of the proposed divider/combiner placed back to back

\section{Conclusion}

A compact four-way power/combiner based on quadruple-ridged waveguide is designed and measured. As well as the compact structure, the proposed power divider/combiner is characterized by its wide bandwidth, high power-combining efficiency, and efficient heat sinking of amplifier chip. As the basic module, this compact quadruple-ridge divider/combiner can be easily extended to multi-way divider/combiner with simple feeding network. 